\title{
ANÁLISE DE VARIÂNCIAS PLUVIOMÉTRICAS NA BACIA HIDROGRÁFICA DO RIO TABOÃO - RS
}

\author{
MARCO ALÉSIO FIGUEIREDO PEREIRA,_MASATO KOBIYAMA, NILZA MARIA DOS REIS \\ CASTRO
}

\author{
Universidade Federal do Rio Grande do Sul (UFRS), Instituto de Pesquisas Hidráulicas, Porto Alegre, RS, \\ Brasil \\ geocram@gmail.com, masato.kobiyama@ufrgs.br, nilza@iph.ufrgs.br
}

Recebido Outubro de 2013 - Aceito Dezembro de 2013

\begin{abstract}
RESUMO
O objetivo deste trabalho foi determinar a distribuição de probabilidade que melhor se ajuste à variância da série histórica de precipitações diárias em seis estações na bacia hidrográfica do rio Taboão, região noroeste do Estado do Rio Grande do Sul, durante um período de sete anos, distribuídos nos intervalos de 05/05/03 a 09/05/06 e 01/07/07 a 04/06/11. Para isso, foram determinados os padrões de agrupamento, em relação à variância. Na comparação do ajustamento das distribuições observadas da variável variância com as distribuições teóricas, foi utilizado o teste de ajustamento de ShapiroWilk. Determinadas as distribuições Log Pearson e Gama, realizou-se o agrupamento das estações de acordo com sua variância pela distância Euclidiana através do método de Ward. Os agrupamentos propostos foram verificados através da ANOVA e teste de Tukey, resultando que os agrupamentos propostos não se sustentavam, demonstrando a homogeneidade entre a média das variâncias das séries históricas das estações analisadas. Os resultados obtidos permitem concluir que a precipitação na bacia do rio Taboão é homogênea.
\end{abstract}

Palavras Chave: Precipitação homogênea, distribuição Log Pearson, distribuição Gama, ANOVA

\begin{abstract}
PRECIPITATION VARIANCE ANALYSIS OF THE TABOÃO RIVER WATERSHED (RS)

The objective of this paper was to determine the probabilistic distribution that fits the variance of the precipitation data obtained in six stations located at Taboão river watershed, northwestern region of Rio Grande do Sul State, during a seven years period from 5/May/2003 to 9/May/2006 and from 1/ July/2007 to 4/June/2011. Group patterns were determined in relation to the variance. The ShapiroWilk test was used for a comparison between the observed and theoretical distributions of the variance. After determining the Log Pearson and Gama distribution, the grouping of stations was performed based on theirs variances by the Euclidean distance using the Ward's method. The evaluation with the ANOVA and Tukey's test showed that the proposed groupings were not sustainable, which implies the homogeneity among the mean values of the historical series variances from the six analyzed precipitation stations. The obtained results permit to conclude that the precipitation is homogeneous in the Taboão river watershed.
\end{abstract}

Keywords: Homogeneous precipitation, Log Pearson distribution, Gama distribution, ANOVA 


\section{INTRODUÇÃO}

O conhecimento da variação da precipitação, tanto no espaço, como no tempo, é importante para estudos hidrológicos e para o planejamento dos recursos hídricos (Bigg, 1991, Sampaio et al., 2007). Por isso, é essencial na implementação de ações políticas, sociais e econômicas para o desenvolvimento sustentável de uma bacia hidrográfica.

Em diversas regiões do Brasil foram realizados alguns trabalhos que identificaram distribuições teóricas que melhor se adequaram a determinadas séries históricas pluviométricas. Por exemplo, Assis (1993) avaliou o ajuste da função Gama em Pelotas-RS; Botelho e Morais (1999) e Morais et al. (2001) avaliaram a distribuição Gama em Lavras - MG; Catalunha et al. (2002) avaliaram cinco funções densidade de probabilidade a séries de precipitação em Minas Gerais; Neto et al. (2005) aplicaram cinco funções densidade de probabilidade a séries pluviométricas em Piracicaba - SP; Sampaio et al. (2007) avaliaram as distribuições decendiais no Estado do Paraná; Souza et al. (2010) compararam as distribuições de probabilidade da precipitação mensal no Estado de Pernambuco; Oliveira et al. (2010) avaliaram a precipitação provável para Alegre - ES através da distribuição de probabilidade Gama.

Embora esses autores tenham analisado as precipitações totais diárias, semanais, quinzenais e mensais, não abordaram a distribuição da variância das precipitações diárias, que é importante saber, pois a variância é diretamente proporcional às oscilações pluviométricas de uma bacia hidrográfica. Nesse sentido Pereira e Kobiyama (2013) realizaram estudo dessa variável na bacia do Alto Cubatão, região metropolitana de Florianópolis-SC.

Diante da necessidade de se conhecer o comportamento da variância pluviométrica na bacia hidrográfica do rio Taboão, que é pertencente à bacia hidrográfica do rio Potiribu, que por sua vez é pertencente à bacia hidrográfica do rio Ijuí - RS, o objetivo do presente trabalho foi determinar a distribuição de probabilidade teórica que melhor se ajusta aos dados de variância da série histórica (05/05/03 a 09/05/06 e 01/07/07 a 04/06/11) e determinar os padrões de agrupamento, em relação à variância, de estações pluviográficas localizadas na bacia do rio Taboão.

Segundo Borges e Bordas (1990) a bacia do rio Ijuí, é representativa de uma área do planalto basáltico sul americano, que abrange $1 \times 10^{6} \mathrm{~km}^{2}$, em termos de relevo, tipo de solo, regime de precipitação, e uso do solo, o qual é essencialmente agrícola.

Desde 1989 o Instituto de Pesquisas Hidráulicas IPH vem desenvolvendo pesquisas hidrológicas nesta bacia, sendo monitoradas diversas variáveis como capacidade de armazenamento e movimento da água no solo, descarga líquida e sólida, sedimentos e qualidade da água (Castro et al., 2000). Mendiondo et al. (2002), abordaram a questão da transferência e correlação de dados de hidrograma em bacias embutidas com áreas de $0,125 \mathrm{~km}^{2}, 1,1 \mathrm{~km}^{2}, 19,9 \mathrm{~km}^{2}, 165 \mathrm{~km}^{2}$, e $569 \mathrm{~km}^{2}$, auxiliados por parcelas experimentais de $1 \mathrm{~m}^{2}$, sob diferentes usos do solo. Silva Junior et al. (2003) avaliaram e compararam a extrapolação espacial na regionalização de vazão nas bacias do rio Ijuí e da bacia do rio Paraopeba - MG, chegando a conclusão que os valores de extrapolação apresentavam menores erros na bacias hidrográficas cujas áreas são maiores que $10 \mathrm{~km}^{2}$. Girardi et al. (2011) avaliaram o efeito de escala em características de chuva e vazão em sub-bacias embutidas da bacia hidrográfica do rio Potiribu. Na mesma bacia Bayer et al. (2012), utilizando modelos ARIMA, analisaram as vazões médias mensais conseguindo modelar e prever as vazões com um período de antecedência de seis meses. Avaliando os trabalhos acima citados, observa-se que na região da bacia do rio Potiribu não foram realizadas pesquisas aprofundando a questão da distribuição da precipitação e sua variância pluviométrica.

\section{MATERIAL E MÉTODOS}

\section{1 Área de estudo}

A bacia hidrográfica do rio Taboão $\left(84 \mathrm{~km}^{2}\right)$ é pertencente à bacia do rio Potiribu $\left(664 \mathrm{~km}^{2}\right)$, esta última ocupa parte dos municípios de Ijuí, Cruz Alta e Pejuçara, região noroeste do Estado do Rio Grande do Sul, situada entre as latitudes $28^{\circ} 23^{\prime} 00^{\prime \prime}$ a $28^{\circ} 28^{\prime} 00^{\prime \prime}$ Se as longitudes $53^{\circ} 35^{\prime} 00^{\prime \prime}$ a $53^{\circ} 47^{\prime} 00^{\prime \prime}$ W (Figura 1). O clima da região está classificado como mesotérmico brando superúmido sem seca $(\mathrm{Cfa})$, pela classificação de Köppen (Beltrame, 2000). As precipitações da região apresentam homogeneidade temporal, sem a presença de uma sazonalidade definida. Girardi et al. (2011) apresentaram as precipitações máximas, médias e mínimas mensais na bacia do Turcato, (bacia inserida na bacia do Taboão) no período de 1990 a 2003 (Figura 2). Os autores afirmaram que a bacia do Turcato apresenta similaridade pluviométrica com a bacia do Taboão, sendo que elas apresentaram a mesma precipitação média mensal $(155 \mathrm{~mm})$ para o referido período.

\subsection{Dados utilizados}

Os dados de precipitação foram coletados em seis pluviógrafos de básculas automáticos, com registro a cada 10 minutos e acumulados a cada hora, com uma precisão de $0,2 \mathrm{~mm}$. A Tabela 1 apresenta algumas informações sobre essas estações pluviográficas. Aqui nota-se que a série histórica dos dados dessas estações é 05/05/03 a 09/05/06 e 01/07/07 a 04/06/11. Assim, tendo dois períodos de observações, encontra-se uma lacuna de um ano e dois meses entre as mesmas. A referida falha ocorreu devido ao não funcionamento dos pluviógrafos existentes na bacia. 


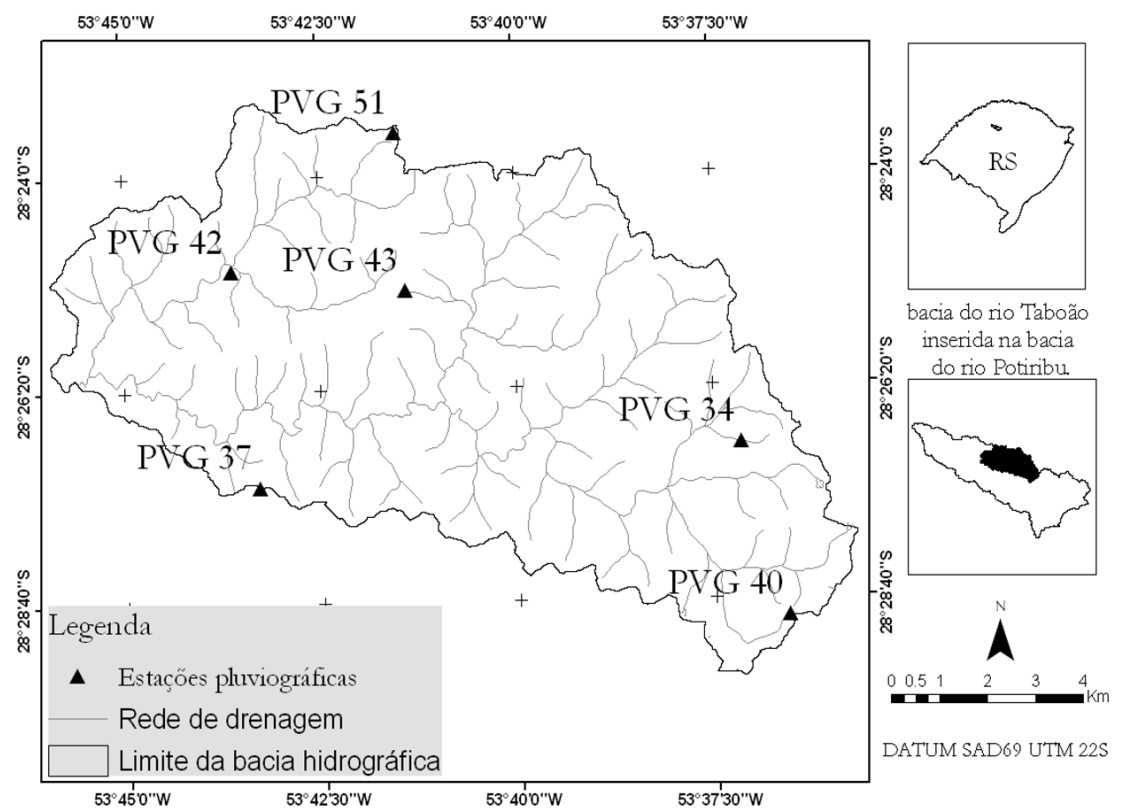

Figura 1 - Localização da bacia do rio Taboão e das estações pluviográficas.

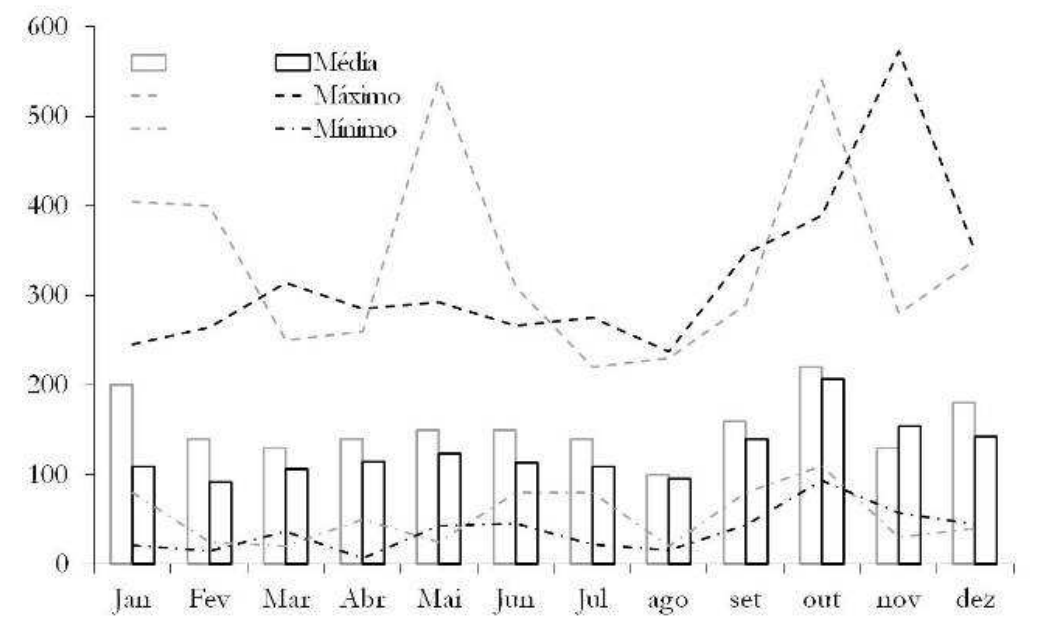

Figura 2 - Precipitações máximas, médias e mínimas da bacia do Taboão (preto) e da bacia do Turcato (cinza). Fonte Girardi et al. (2011) adaptado.

O sistema de armazenamento dos pluviógrafos (PVG 40 e PVG 51) foi realizado através de um registrador eletrônico, modelo LOGBOX. O armazenamento do pluviógrafo PVG 34 foi realizado pelo logger modelo IPH.

Ao final de cada período de tempo, o valor de contagem medido é armazenado na memória do registrador criando uma aquisição. Já os pluviógrafos (PVG 37, PVG 42 e PVG43) utilizaram o registrador eletrônico, modelo HOBO RG3. O procedimento de aquisição e armazenagem dos dados segue o mesmo padrão descrito para os modelos dos loggers anteriores.

Além da falha acima mencionada, no período de 10/05/03 a 30/06/07, existiram pequenos períodos com falhas na série histórica dos diferentes pluviógrafos. Para o preenchimento destas falhas, Depiné et al. (2014) utilizaram a metodologia de Redes Neurais Artificiais (RNA). Para avaliar o desempenho do modelo proposto os autores utilizaram o coeficiente de desempenho de Nash e Sutcliffe (1970), no qual obtiveram resultados que variaram de 0,91 a 0,99 para o treinamento dos modelos e 0,90 a 0,99 para verificação. Após o preenchimento das falhas foram retirados os zeros das séries históricas, sendo trabalhados somente os dias que ocorreram precipitações.

\subsection{Análise estatística}

No presente trabalho as análises estatísticas multivariadas foram realizadas com o programa aplicativo Statistica ${ }^{\circledR}$ 7.0, 
Tabela 1 - Resumo das informações pluviográficas.

\begin{tabular}{llllll}
\hline Estações & Latitudes & Longitudes & Altitude $(\mathrm{m})$ & Pluviógrafo & Logger \\
pluviográficas & & & & & \\
\hline PVG 34 & $28^{\circ} 26^{\prime} 58^{\prime \prime}$ & $53^{\circ} 37^{\prime} 07^{\prime \prime}$ & 440 & Log Chart & IPH \\
PVG 37 & $28^{\circ} 27^{\prime} 23^{\prime \prime}$ & $53^{\circ} 43^{\prime} 17^{\prime}$ & 410 & Water Logger & Hoboware \\
PVG 40 & $28^{\circ} 28^{\prime} 52^{\prime \prime}$ & $53^{\circ} 36^{\prime} 32^{\prime \prime}$ & 470 & Log Chart & LogBox \\
PVG 42 & $28^{\circ} 25^{\prime} 01^{\prime \prime}$ & $53^{\circ} 43^{\prime} 36^{\prime \prime}$ & 384 & Water Logger & Hoboware \\
PVG 43 & $28^{\circ} 25^{\prime} 15$ & $53^{\circ} 41^{\prime} 23$ & 440 & Water Logger & Hoboware \\
PVG 51 & $28^{\circ} 23^{\prime} 32^{\prime \prime}$ & $53^{\circ} 41^{\prime} 29^{\prime}$ & 470 & Log Chart & LogBox \\
\hline
\end{tabular}

desenvolvido pela "StatSoft". Este programa auxilia o usuário na identificação e determinação de parâmetros estatísticos. Para determinar o agrupamento das estações analisadas e chegar a uma conclusão sobre a possível homogeneidade ou heterogeneidade da variância dos dados analisados, foram determinados, antes do teste de ajustamento, os parâmetros de média, mediana, moda, quartil, assimetria e curtose. Também foram avaliados a variância, distribuições teóricas, teste de ajustamento, análise de agrupamento, ANOVA (Análise de Variância) e teste de Tukey. Para exemplificar os passos utilizados para determinar a distribuição de probabilidade que melhor se ajuste à variância da série histórica de precipitações e determinar possíveis padrões de agrupamento, apresenta-se o fluxograma com a metodologia dos procedimentos adotados (Figura 3).

A variância de uma série é a medida de sua dispersão estatística, indicando quão longe em geral os seus valores se encontram do valor esperado (média). Em outras palavras, é o quadrado do desvio padrão, ou seja:

$$
S^{2}=\sum_{i=1}^{n} \frac{\left(x_{i}-\bar{x}\right)^{2}}{n-1}
$$

onde $S$ é o desvio padrão; $n$ é o total de valores analisados; $x_{i}$ é o desvio de cada elemento em relação a média; e $\bar{x}$ é a média.

Com base em Naghettini e Pinto (2007), foram verificadas oito funções de ajustamento (Normal, Log Normal, Gama, Gumbel, Pearson, Log Pearson, Exponencial e T-Student) a fim de avaliar a distribuição teórica que melhor se ajusta a distribuição observada dos dados de variância. Conforme Naghettini e Pinto (2007), as equações das referidas funções são apresentadas na Tabela 2.

\subsection{Teste de ajustamento}

Para averiguar o grau de ajuste dos dados observados à curva teórica de probabilidade ajustada, foi empregado o teste proposto por Shapiro e Wilk (1965).

O referido teste é obtido dividindo o quadrado de uma combinação linear apropriada de uma ordem estatística da amostra pela estimativa simétrica usual da variância.

$$
\begin{aligned}
& S W=\left(\sum_{i=1}^{n} a_{i} \cdot y_{i}\right)^{2} / \sum_{i=1}^{n}\left(y_{i}-\bar{y}\right)^{2} \\
& a_{i}=\sum_{i=1}^{n} m_{i}-v_{i j} / C
\end{aligned}
$$

onde $S W$ é a evidência de quão se ajusta a distribuição hipotética à distribuição observada; $y_{i}$ é o valor da variável; e $\bar{y}$ é a média dos valores das variáveis; $m_{i}=E\left(x_{i}\right) ; v_{i, j}=\operatorname{cov}\left(x_{i}, x_{j}\right)$; e C é um coeficiente linear. Para realização deste teste partisse da premissa que a amostra é uma distribuição normal com média e variância desconhecida. Sendo, portanto, o seu objetivo verificar a veracidade da hipótese nula. Menores valores de $S W$ evidenciam a maior adequação dos dados observados à distribuição teórica.

\subsection{Agrupamento das variáveis}

Para agrupamento das variáveis foi utilizada a técnica de análise de agrupamento, proposta por Scott e Symons (1971), a qual aborda que um conjunto de observações multivariadas são divididas em um número pequeno de grupos relativamente homogêneos. Análise de agrupamento contém uma série de técnicas que visam dividir um conjunto de objetos em diversos 


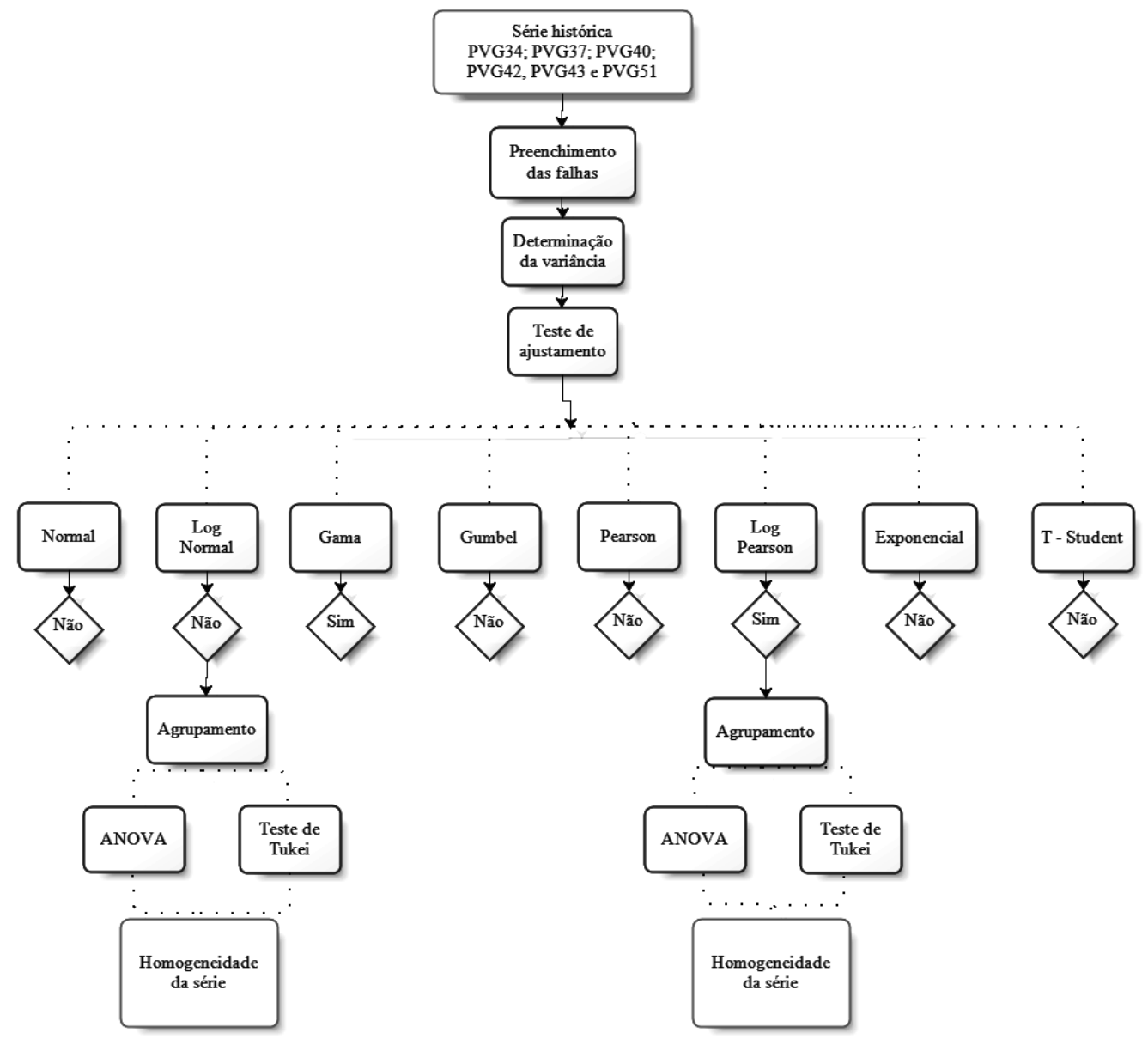

Figura 3 - Fluxograma metodologia adotada.

grupos, onde objetos dentro do mesmo grupo sejam mais semelhantes entre si do que os objetos de grupos diferentes (Bratchell, 1989).

Para determinação dos grupos, utilizou-se a metodologia da distância Euclidiana pelo método de Ward. Conforme Seidel et al. (2008), a distância Euclidiana é a medida de distância mais frequentemente empregada quando todas as variáveis são quantitativas. Tal distância é utilizada para calcular medidas específicas, assim como a distância Euclidiana simples e a distância Euclidiana quadrática ou absoluta, que consiste na soma dos quadrados das diferenças:

$$
D E=\sum_{j=1}^{p}\left(x_{i j}-x_{I j}\right)^{2}
$$

onde $D E$ é a distância euclideana; $x_{i, j}$ é a $j$-ésima característica do $i$-ésimo indivíduo; e $x_{I, j}$ é a $j$-ésima característica do $i$-ésimo indivíduo. Quanto mais próximo de zero for $D E$, mais similares são os objetos comparados.

No método de Ward Júnior (1963), a distância entre dois agrupamentos é a soma dos quadrados entre os dois agrupamentos feita sobre todas as variáveis. Nesse método a formação dos grupos se dá pela maximização da homogeneidade dentro dos grupos.

$$
W=\sum_{i=1}^{n} X_{i}^{2}-\frac{1}{n}\left(\sum X_{i}\right)^{2}
$$

onde $W$ apresenta a homogeneidade intra-grupos e heterogeneidade inter-grupos através da soma do quadrado dos desvios; $n$ é o número de valores analisados; e $X_{i}$ é o $i$-ésimo elemento do agrupamento. Este método tem se revelado um dos melhores e mais usados métodos hierárquicos de aglomeração (Kubrusly, 2001; Seidel et al., 2008).

\subsection{ANOVA}

A ANOVA tem por objetivo determinar se todos os grupos têm valores médios idênticos, comparando duas estimativas de variância total. Para a hipótese nula (H0) ser verdadeira, a média dos grupos deverá ser muito similar à variância dentro de um grupo. Se a média dos grupos é 
Tabela 2 - Distribuições teóricas aplicadas para tentativa de ajustamento aos dados.

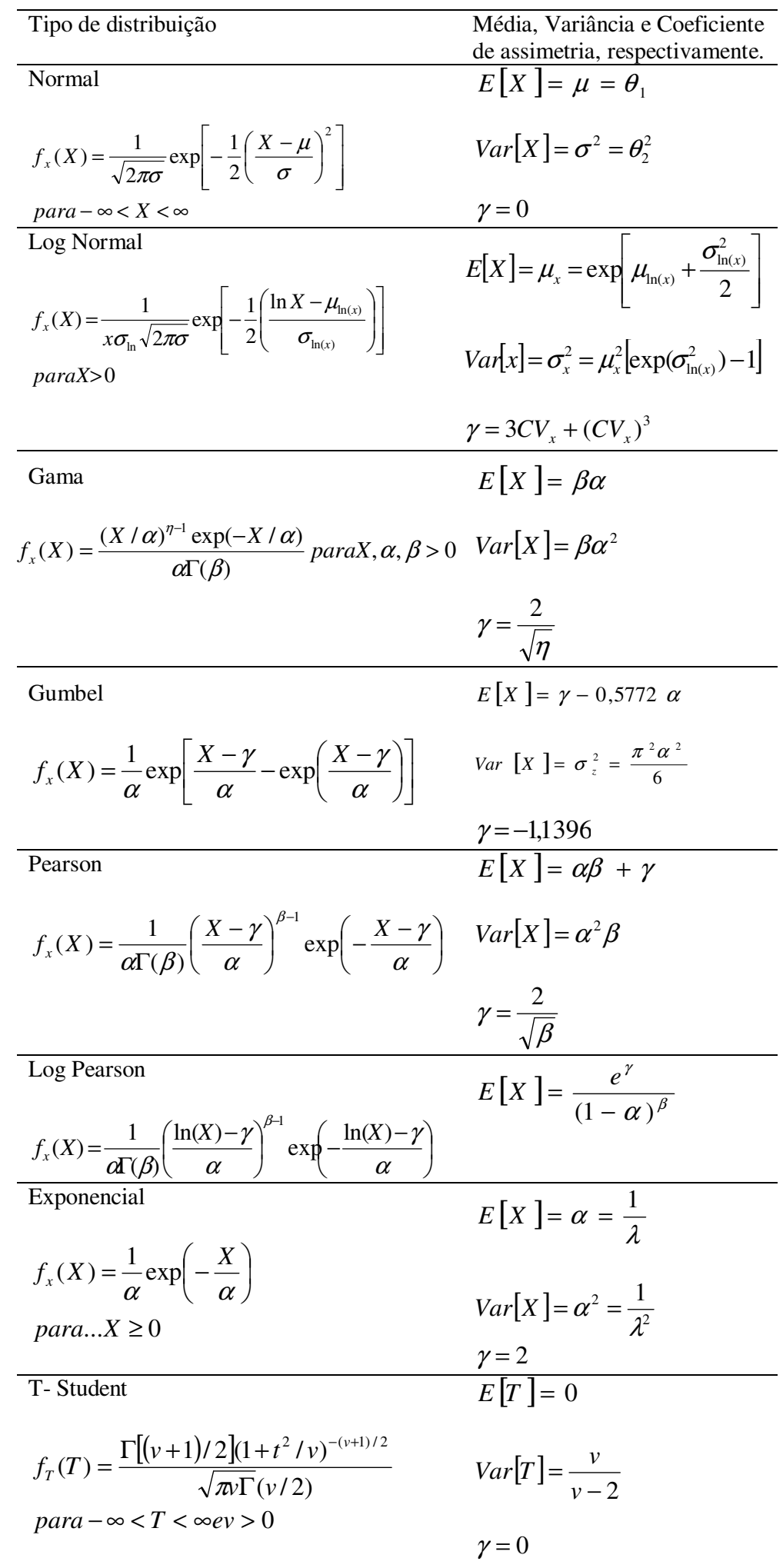

Obs: $X$ é a variável aleatória; $\mu$ é a média; $\sigma$ o desvio padrão; $\alpha$ parâmetro de escala; $\beta$ parâmetro de forma; $\gamma$ é o parâmetro de posição, $\lambda$ o parâmetro utilizado para normalização; $T$ é a variável aleatória; e $v=n$-1 graus de liberdade. 
diferente, alguns deles serão suficientemente diferentes da média geral, sendo a variância dentro dos grupos não mais igual à variância total. A variância dentro dos grupos é calculada pela estimativa do erro quadrado médio (Hirsch et al., 1992):

$$
M S E=\frac{\sum_{j=1}^{k} \sum_{i=1}^{n_{j}}\left(y_{i j}-\bar{y}_{j}\right)}{N-K}
$$

onde $M S E$ é o erro quadrado médio; $y_{i j}$ é o valor observado no grupo; $\bar{y}_{j}$ é a média do $j$-ésimo grupo; $N$ é o número de observações em cada grupo; e $K$ é o número de grupos.

\subsection{Teste de Tukey}

O teste proposto por Tukey (1949) também conhecido como teste da diferença honestamente significativa (honestly significant difference - HSD) consiste em definir a menor diferença significativa entre a média de dois grupos. Tal procedimento utiliza a amplitude da distribuição T-student e geralmente trabalha com um nível de significância de 1 ou $5 \%$. Quando $n$ for igual para as duas séries de dados utiliza-se a Equação 7, quando $n$ for diferente usa-se a expressão 8 (Montgomery e Runger, 2003).

$$
\begin{aligned}
& H S D=g_{\alpha}(a, f) \sqrt{\frac{M S E}{n}} \\
& H S D=\frac{g_{\alpha}(a, f)}{\sqrt{2}} \sqrt{\left(\frac{1}{n_{1}} \cdot \frac{1}{n_{2}}\right) \cdot M S E}
\end{aligned}
$$

onde $H S D$ é o valor crítico para a diferença entre as médias dos grupos; $g_{\alpha}(a, f)$ é o ponto da percentagem superior de $\alpha$ do intervalo da estatística studentizada; $a$ é o número de tratamentos; $f$ é o número de graus de liberdade; e $n_{1}$ e $n_{2}$ são os números de amostras de cada série analisada. Nota-se que $g_{\alpha}(a, f)$ são valores tabelados.

\section{RESULTADOS E DISCUSSÕES}

\subsection{Variância}

Na Figura 4 é demonstrado o período sem dados de precipitação na bacia em estudo. Com os dados de $S^{2}$, determinados através da Equação 1, pode-se inferir outras variáveis relacionadas a $S^{2}$ das séries históricas, estes dados são apresentados na Tabela 3

Para determinar a distribuição que melhor se ajusta aos dados de $\mathrm{S}^{2}$ das estações pluviográficas, localizadas na bacia do Taboão, foi realizado o teste de Shapiro e Wilk à $5 \%$ de significância. A Tabela 4 apresenta os resultados do respectivo teste de ajustamento para os dados teóricos e observados. Salienta-se que neste teste, quanto mais próximo a zero for à inferência, maior será a hipótese que a distribuição teórica se ajusta a distribuição observada.

Para o teste SW observa-se que nas distribuições Log Normal, Gumbel e T-Student houve aumento na inferência do teste em todas as séries. A distribuição Normal também demonstrou aumento na inferência em todas as séries, com exceção da série PVG 40. Assim, configurando a rejeição da hipótese nula, ou seja, a distribuição teórica não se ajusta para representar a distribuição observada.

As distribuições Log Pearson, Gama, Exponencial, e Pearson, em ordem de citação, demonstraram melhor desempenho na inferência de ajuste entre a série teórica e a observada. Diante desta constatação, concluí-se que as funções

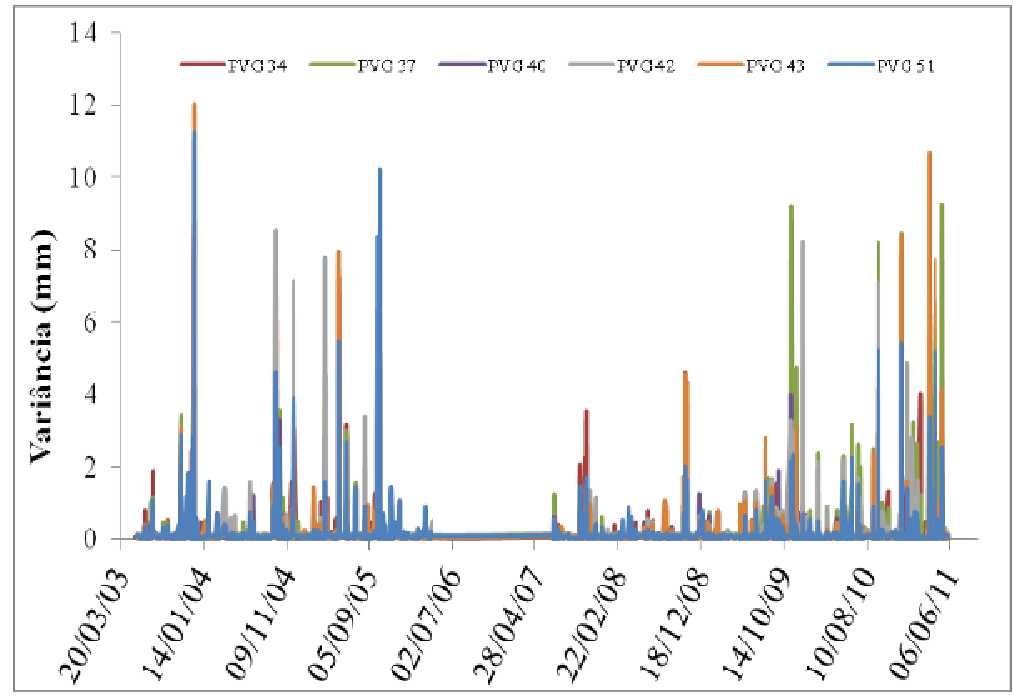

Figura 4 - Dispersão de $\mathrm{S}^{2}$ no tempo. 
Tabela 3 - Estatística das precipitações diárias.

\begin{tabular}{cccccccccc}
\hline Estação & $\mathrm{N}$ & $\begin{array}{c}\text { Média } \\
(\mathrm{mm} / \mathrm{dia})\end{array}$ & $\begin{array}{c}\text { Mediana } \\
(\mathrm{mm} / \mathrm{dia})\end{array}$ & $\begin{array}{c}\text { Moda } \\
(\mathrm{mm} / \mathrm{dia})\end{array}$ & $\begin{array}{c}\text { Frequência } \\
\text { da moda }\end{array}$ & $25 \%$ Quartil & $75 \%$ Quartil & Assimetria & Curtose \\
\hline PVG34 & 1059 & 0,24 & 0,10 & 0,10 & 219 & 0,05 & 0,10 & 6,87 & 57,76 \\
PVG37 & 1032 & 0,28 & 0,10 & 0,11 & 172 & 0,05 & 0,11 & 6,72 & 52,82 \\
PVG40 & 1160 & 0,15 & 0,05 & 0,05 & 227 & 0,03 & 0,06 & 7,96 & 81,61 \\
PVG42 & 1067 & 0,24 & 0,09 & 0,09 & 203 & 0,04 & 0,09 & 7,72 & 71,52 \\
PVG43 & 1128 & 0,18 & 0,06 & 0,06 & 188 & 0,03 & 0,06 & 10,23 & 127,05 \\
PVG51 & 1048 & 0,20 & 0,07 & 0,07 & 160 & 0,03 & 0,08 & 9,43 & 111,86 \\
\hline
\end{tabular}

Teste de ajustamento

Tabela 4 - Teste de ajustamento com teste de Shapiro e Wilk.

\begin{tabular}{|c|c|c|c|c|c|c|c|c|c|c|c|c|}
\hline \multirow{2}{*}{ Distribuição } & \multicolumn{2}{|c|}{ PVG 34} & \multicolumn{2}{|c|}{ PVG 37} & \multicolumn{2}{|c|}{ PVG 40} & \multicolumn{2}{|c|}{ PVG 42} & \multicolumn{2}{|c|}{ PVG 43} & \multicolumn{2}{|c|}{ PVG 51} \\
\hline & Observada & Teórica & Observada & Teórica & Observada & Teórica & Observada & Teórica & Observada & Teórica & Observada & Teórica \\
\hline Normal & 0.40 & 0.48 & 0.41 & 0.49 & 0.40 & 0.09 & 0.39 & 0.51 & 0.40 & 0.55 & 0.40 & 0.52 \\
\hline Log Normal & 0.40 & 0.70 & 0.41 & 0.67 & 0.40 & 0.80 & 0.39 & 0.69 & 0.40 & 0.75 & 0.40 & 0.73 \\
\hline Gama & 0.40 & 0.10 & 0.41 & 0.09 & 0.40 & 0.12 & 0.39 & 0.10 & 0.40 & 0.12 & 0.40 & 0.09 \\
\hline Gumbel & 0.40 & 0.44 & 0.41 & 0.65 & 0.40 & 0.52 & 0.39 & 0.46 & 0.40 & 0.52 & 0.40 & 0.48 \\
\hline Log Pearson & 0.40 & 0.09 & 0.41 & 0.12 & 0.40 & 0.10 & 0.39 & 0.09 & 0.40 & 0.10 & 0.40 & 0.08 \\
\hline Exponencial & 0.40 & 0.17 & 0.41 & 0.19 & 0.40 & 0.22 & 0.39 & 0.19 & 0.40 & 0.23 & 0.40 & 0.19 \\
\hline T - Student & 0.40 & 0.49 & 0.41 & 0.49 & 0.40 & 0.57 & 0.39 & 0.51 & 0.40 & 0.56 & 0.40 & 0.53 \\
\hline
\end{tabular}

Log Pearson e Gama são as distribuições de probabilidade que melhor representam os dados das séries históricas em análise. Portanto, o presente trabalho escolheu essas duas distribuições que podem ser usadas para identificar os possíveis agrupamentos de $S^{2}$.

\subsection{Agrupamento}

Para apresentar o possível agrupamento proposto pelo método de análise de agrupamento das variáveis, pelas distribuições Log Pearson e Gama, utiliza-se o dendograma com dois e três grupos (Figura 5).

$\mathrm{Na}$ separação em dois grupos observa-se o mesmo resultado de agrupamento para as duas distribuições. Quando se separa os grupos em três grupos distintos observa-se uma diferença entre as distribuições. Na distribuição Log Pearson a série PVG 37 forma um único grupo, enquanto na distribuição Gama esta série forma grupo com a série PVG 51.

\subsection{ANOVA}

Para verificar a consistência do agrupamento proposto pelo método de análise de agrupamento foi realizada a ANOVA. Esta análise tem como objetivo comprovar as diferenças entre os grupos através da homogeneidade das variâncias das médias dentro de cada grupo (whitin) pelos gráficos das variâncias, para dois e três grupos, (Figuras 6 e 7) e a independência dos grupos através da verificação de diferenças significativas entre as médias de cada grupo pelo teste HSD (Tabelas 5 e 6).

Aplicando-se a Equação 7, com $\alpha=5 \%$ verifica-se o valor de $\mathrm{HSD}=0,0103$ para dois grupos e $\mathrm{HSD}=0,0187$ para três grupos na a distribuição Log Pearson. No caso da distribuição Gama, os valores de HSD são 0,0135 e 0,0187 para dois grupos e três grupos, respectivamente. O objetivo desta análise é verificar a homogeneidade dos grupos propostos pela distância Euclidiana pelo método de Ward (Figuras 6 e 7). Para que a hipótese nula (grupos iguais) seja aceita, admite-se que não existem diferenças significativas entre as médias. Portanto, os valores de HSD devem ser menores que os valores encontrados nos agrupamentos propostos (Tabelas 5 e 6 ).

Seguindo esta metodologia de análise, constata-se que os agrupamentos propostos, tanto em dois, quanto em três grupos devem ser rejeitados. Diante destas constatações comprova-se que a análise da variância das precipitações medidas nas estações pluviográficas da bacia do rio Taboão apresenta similaridade, configurando o aceite da hipótese nula. Isto implica homogeneidade hidrológica para esta variável, no período em análise.

Esta homogeneidade hidrológica para $S^{2}$ pode ser explicada em virtude das características geomorfológicas da região, pois como Borges e Bordas (1990) comentaram, a região da bacia do rio Taboão apresenta a homogeneidade em termos de relevo. 
a
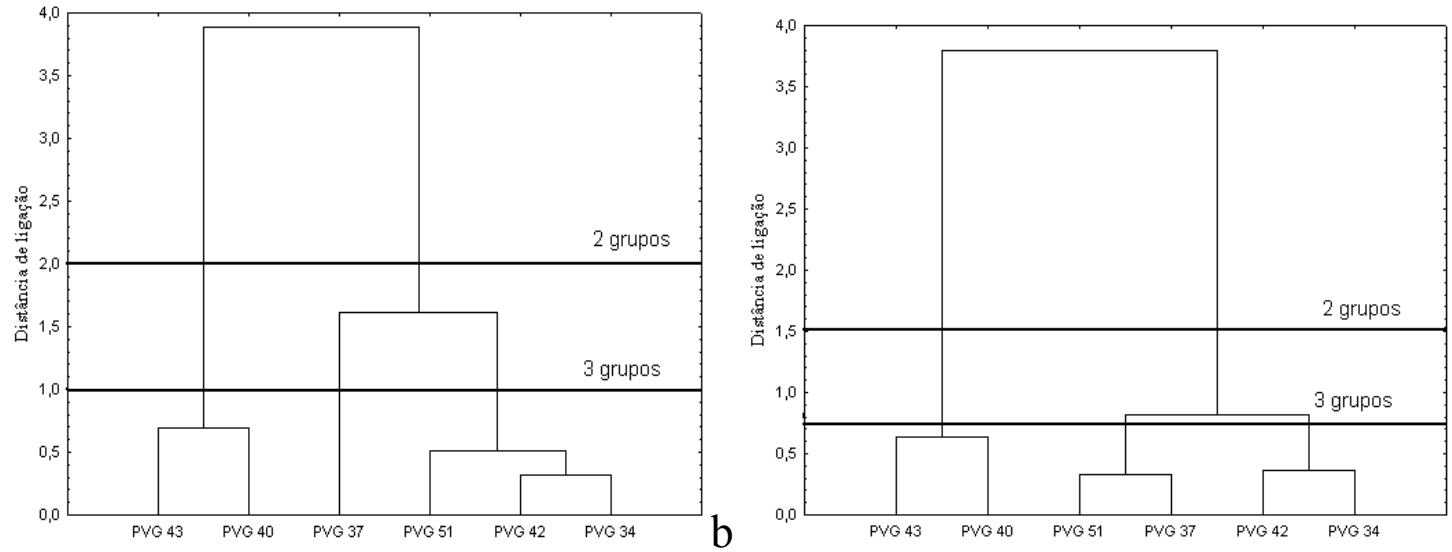

Figura 5 - Dendograma para o agrupamento da variância das estações analisadas. (a) Log Pearson e (b) Gama
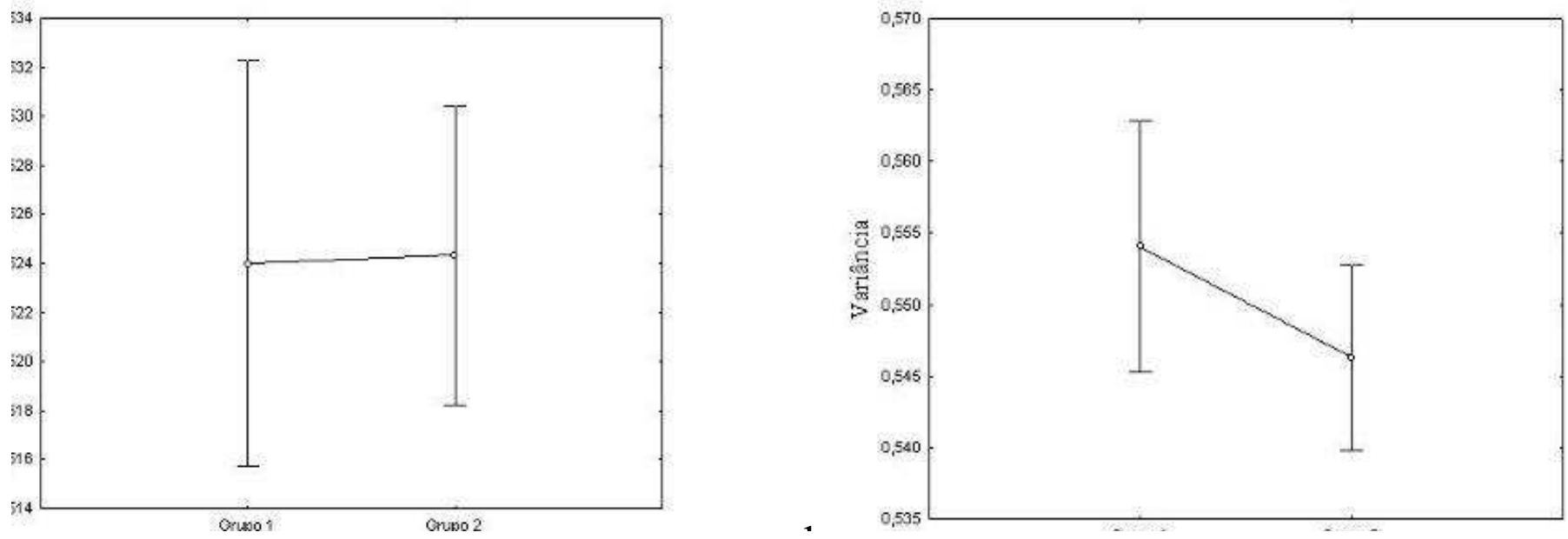

Figura 6 - Teste de homogeneidade pelos gráficos de médias e variâncias: (a) Log Pearson e (b) Gama.
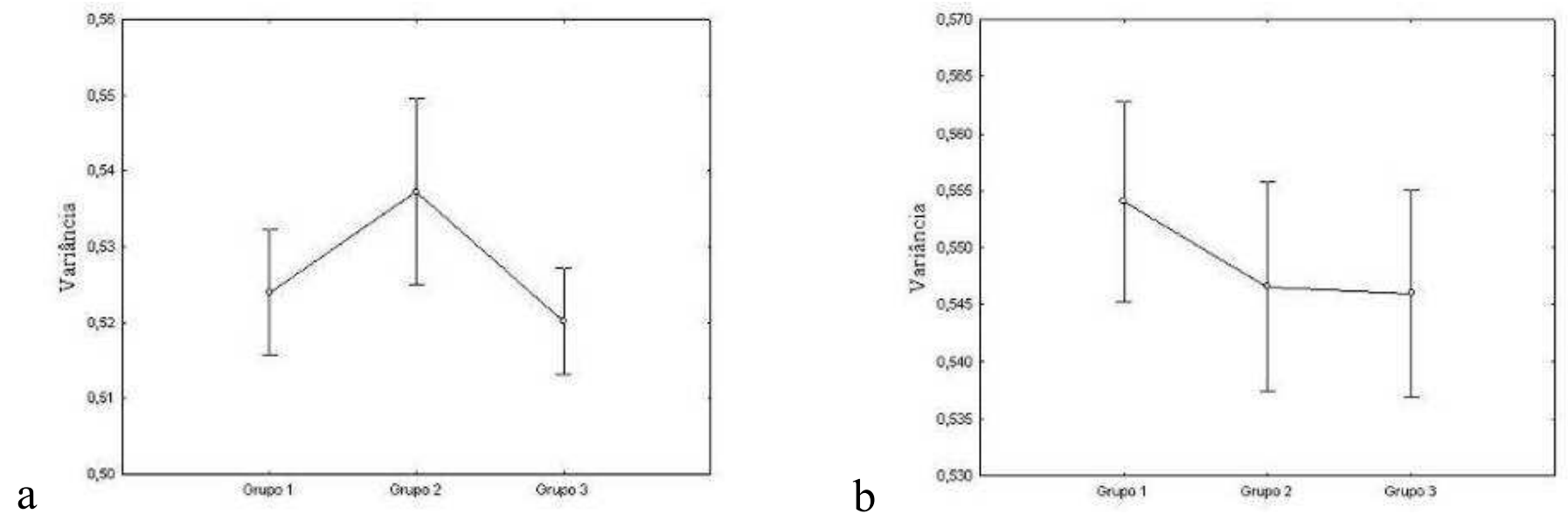

Figura 7 - Teste de homogeneidade pelos gráficos de médias e variâncias: (a) Log Pearson e (b) Gama. 
Tabela 5 - Teste de independência de HSD para Log Pearson e Gama.

Teste HSD para Log Pearson

Homogeneidade do grupo $\alpha=0,05$

Erros entre MSE $=0,04074 \mathrm{df}=6492$

\begin{tabular}{cccc}
\hline Grupo & Média & $(1)$ & $(2)$ \\
\hline 1 & 0,5240 & & 0,9502 \\
2 & 0,5243 & 0,9502 & \\
\hline
\end{tabular}

Teste HSD para Gama

Homogeneidade do grupo $\alpha=0,05$

Erros entre MSE $=0,04601 \mathrm{df}=6492$

\begin{tabular}{cccc}
\hline Grupo & Média & $(1)$ & $(2)$ \\
\hline 1 & 0,5540 & & 0,1621 \\
2 & 0,5462 & 0,1621 & \\
\hline
\end{tabular}

\section{CONCLUSÕES}

A fim de compreender melhor a hidrologia da bacia do rio Taboão - RS, o presente trabalho analisou os dados de precipitação das seis estações instaladas na mesma, e determinou a distribuição de probabilidade que melhor se ajustou à variância da série histórica pluviométrica e definiu os padrões de agrupamento, em relação à variância.

Com a aplicação do teste de Shapiro e Wilk para as distribuições teóricas em análise constata-se que a distribuição Log Pearson e Gama apresentaram melhor ajustamento aos dados observados. Portanto, as respectivas distribuições se ajustam para representar os dados de $\mathrm{S}^{2}$ em análise. Quando se realizou o agrupamento da variância pela distância Euclidiana pelo método de Ward foi definido que as variáveis se enquadravam em dois e três grupos. Porém, com a aplicação da ANOVA e do teste HSD, verificou-se que os possíveis agrupamentos das variáveis não se aplicavam, demonstrando, portanto, a importância da aplicação da ANOVA e teste HSD para verificação dos agrupamentos sugeridos.

Analisando os referidos testes, concluiu-se que a variância da precipitação na bacia hidrográfica do rio Taboão apresenta homogeneidade espacial. Para finalizar, recomenda-se a continuidade do monitoramento da precipitação na bacia e a elaboração de outros trabalhos que venham a investigar a variância numa escala espaço-temporal maior.

\section{REFERÊNCIAS}

ASSIS, F.N.; Ajuste da função gama aos totais semanais de chuva de Pelotas-RS. Revista Brasileira de Agrometeorologia, Santa Maria, v. 1, p. 131-136, 1993.
Tabela 6 - Teste de independência de HSD para Log Pearson e Gama.

\begin{tabular}{ccccc}
\hline \multicolumn{5}{c}{ Teste HSD para Log Pearson } \\
\multicolumn{5}{c}{ Homogeneidade do grupo $\alpha=0,05$} \\
Erros entre MSE $=0,04602 \mathrm{df}=6491$ \\
\hline Grupo & Média & $(1)$ & $(2)$ & $(3)$ \\
1 & 0,5240 & & 0,188 & 0,764 \\
2 & 0,5372 & 0,188 & & 0,047 \\
3 & 0,5201 & 0,764 & 0,047 & \\
\hline \multicolumn{5}{c}{ Teste HSD para Gama } \\
\multicolumn{5}{c}{ Homogeneidade do grupo $\alpha=0,05$} \\
\hline Grupo & Média & $(1)$ & $(2)$ & $(3)$ \\
1 & 0,5540 & 0,480 & 0,424 \\
2 & 0,5465 & 0,480 & 0,995 \\
3 & 0,5460 & 0,424 & 0,995 \\
\hline
\end{tabular}

BAYER, D. M.; CASTRO, N. M. dos R.; BAYER, F. M.; Modelagem e Previsão de Vazões Médias Mensais do rio Potiribu utilizando modelos de séries temporais. Revista Brasileira de Recursos Hídricos, v. 17 n. 2, pp. 229-239, 2012. BELTRAME, L. F. de S. (coord.). Consistência de Dados Hidrológicos da Bacia Hidrográfica do Alto Uruguai, Sub-Bacia 75. Porto Alegre, Instituto de Pesquisas Hidráulicas da UFRGS, 2000.

BIGG, G.R.; Kriging and intraregional rainfall variability in England. International Journal of Climatology v.11, p.663-675, 1991.

BORGES, A.L.; BORDAS, M.P.; Escolha de bacias representativas e experimentais para estudo da erosão no planalto basáltico sulamericano. Congresso Brasileiro e Encontro Nacional de Pesquisas sobre Conservação de Solo, 1990, Londrina, Anais.

BOTELHO, V. A.; MORAIS, A. R.; Estimativas dos parâmetros da distribuição gama de dados pluviométricos do Município de Lavras, Estado de Minas Gerais. Ciência e Agrotecnologia, Lavras, v. 23, p. 697-706, 1999.

BRATCHELL, N.; Cluster Analysis. Chemometrics and Intelligent Laboratory Systems, v. 6, pp. 105-125, 1989.

CATALUNHA, M. J.; SEDIYAMA, G. C.; LEAL, B. G.; SOARES, C. P.; RIBEIRO, A. B.; Aplicação de cinco funções densidade de probabilidade a séries de precipitação pluvial no Estado de Minas Gerais. Revista Brasileira de Agrometeorologia, Santa Maria, v. 10, n. 1, p. 153-162, 2002.

CASTRO, N. M. dos R.; CHEVALLIER, P.; GOLDENFUM, J.; Recursos Hídricos. Projeto Potiribu - Atualização 19891998 - Dados básicos de fluviometria e pluviometria. Ministério da Educação UFRGS-IPH, v. 35, 65p., 2000. 
DEPINÉ, H.; CASTRO, N. M. dos R.; PEDROLLO, O.; PINHEIRO, A.; Preenchimento de falhas de dados horários de precipitação utilizando redes neurais artificiais. Revista Brasileira de Recursos Hídricos, v. 19 n.1, pp. 51-63, 2014. GIRARDI, R.V.; CASTRO, N. M. dos R.; GOLDENFUN, J. A.; SILVEIRA, A. L. L. da; PINHEIRO, A.; Avaliação do Efeito de Escala em Características de Chuva e Vazão em Sub-Bacias Embutidas da Bacia do Potiribu - RS. Revista Brasileira de Recursos Hídricos, v.16 n.2, pp. 49-64, 2011. HIRSCH, T. A.; HESEL, D. R.; COHN, T. A.; GILROY, E. J.; Statistical treatment of data of hydrologic data. (cap. 17) Handbook of hydrology. U.S. Geological Survey. Ed. Donnelley\&Sons Company. New York, p. 645-696, 1992.

KUBRUSLY, L. S.; Um procedimento para calcular índices a partir de uma base de dados multivariados. Pesquisa Operacional, v.21 n.1, p.107-117, 2001.

MENDIONDO, E. M. ; TUCCI, C. E. M.; CLARKE, R. T.; CASTRO, N. M.; GOLDENFUM, J.; CHEVALLIER, P.; Space-time observations in nested catchment experiments of representative basins - experiences gained and lessons learned to help the PUB initiative in the World's biomes. Predictions in Ungauged Basins: PUB Kick-off Meeting (2002: Brasília), 2007, Brasília. Proceedings, Wallingford: IAHS, 2007. Wallingford : IAHS, v. 1. p. 164-172, 2002.

MONTGOMERY, D.C.; RUNGER, G.C.; Applied statistic and probability for engineers. 3rd ed. United States of America. p. 822, 2003.

MORAIS, A. R. DE; BOTELHO, V. A. V. AMARANTE; CARVALHO, L. G. DE; MUNIZ, J.AUGUSTO; LAGE, GILBERTO.; Estimativa da precipitação provável em Lavras (MG) através da distribuição gama. Revista Brasileira de Agrometeorologia, Santa Maria, v. 9, n. 2, p. 305-310, 2001.

NAGHETTINI, M. PINTO, É. J. de A.; Hidrologia Estatística. Belo Horizonte, CPRM, 552p., 2007.

NASH, J. E.; SUTCLIFFE, J. V.; River flow forecasting through conceptual models, Part 1: A discussion of principles. Journal of Hydrology, v. 10, n. 3, p. 282-290, 1970.

NETO, D. D.; ASSIS, J. P. DE; TIMM, L. C.; MANFRON, P. A.; SPAROVEK, GERD; MARTIN, T. N.; Ajuste de modelos de distribuição de probabilidade a séries históricas de precipitação pluvial diária em Piracicaba - SP. Revista Brasileira de Agrometeorologia, v. 13 n.2, p. 273-283, 2005.
OLIVEIRA, J. P. B. de; CECÍlIO, R. A.; XAVIER, A. C.; JASPER, A. P. S.; OLIVEIRA, L. B.; Precipitação provável para Alegre-ES através da distribuição de probabilidade Gama. In. Engenharia Ambiental - Espírito Santo do Pinhal, v. 7 n. 2, p.204-211, 2010.

PEREIRA, M. A. F.; KOBIYAMA, M.; Análise de variâncias pluviométricas na região da bacia hidrográfica do Cubatão do Sul - SC. Ambiência, Guarapuava (PR) v. 9 n.1 p. 95 $-111,2013$.

SAMPAIO, S. C.; QUEIROZ, M. M. F. de; FRIGO, E. P.; LONGO, A. J.; SUSZEK, M.; Estimativa e distribuição de precipitações decendiais para o Estado do Paraná. Irriga, Botucatu, v. 12 n. 1, p. 38-53. 2007.

SCOTT, A. J.; SYMONS, M. J.; On the Edwards and CavalliSforza Method of Cluster Analysis. Biometrics. Vol27. $\mathrm{N}^{\circ} 1$, University of North Carolina, Chapel Hill, U.S.A., p.217-219, 1971.

SEIDEL, E. J.; MOREIRA JÚNIOR, F. de J.; ANSUJ, A. P.; NOAL, M. R.; Comparação entre o método Ward e o método K-médias no agrupamento de produtores de leite. Ciência e Natureza, v.30, n.1, p.7- 15, 2008.

SHAPIRO, S.S.; WILK, M. B.; An Analysis of Variance Test for Normality (Complete Samples). Biometrika, v. 52, pp. 591-611, 1965.

SILVA JUNIOR, O. B. ; BUENO, E. de O.; TUCCI, C. E. M. ; CASTRO, N. M. R.; Extrapolação Espacial na Regionalização da Vazão. Revista Brasileira de Recursos Hídricos v.8 n.1, pp 21-37, 2003.

SOUZA, I. F.; LUNDGREN, W. J. C.; NETTO, A. O. A.; Comparação entre distribuições de probabilidade da precipitação mensal no Estado de Pernambuco. Scientia Plena, v.6 n.6, 10p., 2010.

TUKEY, J. W.; Comparing Individual Means in the Analysis of Variance, Biometrics, v.5, p. 99. 1949.

WARD JÚNIOR, J. H.; Hierarchical grouping to optimize an objective function. Journal of the American Statistical Association, v.58 n.301, p.236-244, 1963. 